\title{
Emergent dynamical features in behaviour-incidence models of vaccinating decisions
}

\author{
Samit Bhattacharyya and Chris T. Bauch
}

\begin{abstract}
Vaccination is a cornerstone of infectious disease prevention. However, individual vaccinating behaviour does not always result in population-level vaccine coverage patterns that are optimal for protecting public health. For example, vaccine coverage may fall below the elimination threshold due to nonvaccinators who "freeride" on the herd immunity provided by vaccinators. Routine vaccination programs for many pediatric infectious diseases now have an almost world-wide coverage, but vaccine scares fuelled by such behaviours threaten eradication goals. This freeriding behaviour can be seen as a manifestation of policy resistance, where humans respond to an intervention in such a way that tends to undermine the intervention. However, policy resistance is only one such example of the types of dynamics that emerge from the interaction between vaccinating behaviour and disease incidence or prevalence. Here we explore four types of emergent dynamics of behaviour-incidence systems: policy resistance, policy reinforcement, outcome inelasticity, and outcome variability. We discuss examples of each of these dynamics in the behaviour-incidence modelling literature, and suggest potential implications for vaccination policy.
\end{abstract}

\section{Introduction}

Despite widespread controversies among the public, vaccination has proved to be one of the most successful infectious disease interventions ever, and remains one

Samit Bhattacharyya

Departments of Mathematics and Biology, School of Medicine, University of Utah, Salt Lake City, UT 84108, e-mail: samit@math.utah.edu

Chris T. Bauch

Department of Mathematics and Statistics, University of Guelph, 50 Stone Road, Guelph, ON N1G2W1, e-mail: cbauch@uoguelph.ca 
of the greatest public health achievements in the twenty-first century. Vaccination against major infectious diseases, and the complete or near-complete eradication of some diseases (such as smallpox and polio) has completely changed the demography of many developed and developing countries worldwide [13].

The most common strategy is universal mass vaccination (UMV), which requires covering as much of the population as possible, either through large-scale periodic campaigns or through regular school-based programs. However, ring vaccination has also been applied. Ring vaccination involves identifying infectious index cases and vaccinating their close contacts to prevent them from being infected [37, 29]. Ring vaccination may perform better than UMV when the outbreak is localized and infected individuals or their exposed contacts can be rapidly identified. Ring vaccination has been applied to outbreak control for hepatitis A [17], foot-and-mouth disease in cattle [32, 41] and smallpox [21].

Large-scale vaccination programs confers population-wide benefits. Vaccination not only prevents infection in vaccinated individuals-it also protects the unvaccinated through a "herd immunity" effect that slows down the circulation of a pathogen in the entire population [1]. Herd immunity operates through disrupting the chain of transmission between individuals. The greater the proportion of vaccinated individuals, the smaller the probability that a susceptible individual will come into contact with an infectious individual and thereby become infected.

The epidemiology of many well-known vaccine preventable diseases is subject to the effects of human belief and awareness of disease or vaccine [15, 22]. Human behaviour plays an important role in determining whether target vaccination coverage can be reached in a given population. Common childhood diseases such as measles or pertussis are timely examples [38]. While there has long been enthusiastic debate in some higher-income countries about the relative merits and implications of mandatory versus voluntary vaccination [16], it is clear that voluntary vaccination policies sometimes fail. Measles-Mumps-Rubella (MMR) vaccination in Great Britain in the 1990s is one such example of failure due to a vaccine "scare" [31].

Vaccination coverage for seasonal influenza also remains suboptimal in many countries, including the USA and Canada. Sub-optimal coverage has been observed even among health care workers (HCWs). For example, a recent study indicates that vaccination coverage for seasonal influenza among HCWs in Canada remains below 50\% [33]. Influenza vaccination coverage among children and individuals at high-risk was also very low and significantly below the target level. This is a worldwide phenomenon, with suboptimal influenza vaccine coverage among HCWs having also been identified in Middle East countries (United Arab Emirates (UAE), Kuwait and Oman), due to doubts about vaccine efficacy, lack of information about the importance of immunization, and concerns about vaccine side effects [28]. A low perceived risk of becoming infected, whether justified by historically low infection rates or not, can contribute as much as inflated perception of vaccine risk does: studies identify perceived lack of infection risk as a factor in non-uptake of influenza vaccine [35]. If reduced infection rates due to previous vaccinations lead to reduced perception of infection risks, then herd immunity can, ironically, lead to reduced infection risk perception and thus reduced vaccine uptake. 
An emerging fear of vaccine complications can combine with the temptation to rely on herd immunity provided by those who have already vaccinated to impel individuals to exempt themselves or their children from vaccination. Thus, herd immunity introduces a social dilemma in voluntary vaccination policy that amounts to "free-riding", or a "Tragedy of the Commons" [30, 22]. Equivalently, this dynamic implies a feedback loop between vaccinating decisions and disease dynamics: individual vaccinating choices influence disease prevalence, but the level of disease prevalence in turn influences how many individuals choose to seek vaccination [7, 9, 11] (Figure 1).

Classical game theory provides a useful tool to analyze and predict the outcomes of strategic interactions [44, 43, 20,18], including those arising from the interaction between disease dynamics and human vaccinating behaviour [14, 5]. According to a game theoretical perspective, individuals make a rational decision in weighing up the costs and benefits related to vaccination against the cost of risking infection, making assumptions about how much herd immunity will be provided by others in the population. Although further empirical study is warranted regarding how well game theory captures vaccinating behaviour and risk perception, game theoretic modeling of individual vaccinating decisions is growing. This models often predict that rational self-interest leads to a Nash equilibrium vaccine coverage that is suboptimal for the population, being below the level required to eliminate the infection $[6,5]$. However, a variety of other non-game-theoretical approaches have also been adopted to capture the interplay between disease dynamics and vaccinating behaviour, and they often yield similar predictions $[2,7,19]$.

The nonlinear feedback loop that springs from interaction between individual vaccinating behaviour and disease dynamics can create interesting dynamical consequences, including policy resistance, policy reinforcement, outcome inelasticity, and outcome variability. In the next few sections, we will define these terms and discuss how they arise in models of the interplay between vaccinating behaviour and disease dynamics.

\section{Policy resistance}

The most common implication of herd immunity for behaviour-incidence dynamics is policy resistance, which is "the tendency for interventions to be defeated by the systems response to the intervention itself" [40]. The vaccine coverage necessary to achieve perfect herd immunity and thus elimination varies greatly from one infectious disease to the next, but generally ranges from $80 \%-95 \%$ for common pediatric infectious diseases [1]. If this level of vaccination is attained, those who refuse to be vaccinated are nonetheless protected through the strong likelihood that they will never be infected. As a result, there is a temptation not to seek vaccination, when vaccine coverage is very high. If this temptation translates into individual action not to seek vaccination, vaccine coverage will drop below the elimination threshold. Hence, as a result of herd immunity and the nonlinear interplay between disease 
dynamics and vaccinating behaviour, voluntary vaccination is subject to policy resistance.

Policy resistance is often cast as a conflict between the Nash equilibrium vaccine coverage and the social optimum vaccine coverage. The social optimum can be defined as the vaccine coverage such that the total population burden from either vaccination or infection across all individuals is minimized, and in these models the goal of public health is often conceived as being to reach the socially optimal coverage level (although typically, this definition ignores issues of equity). In contrast to the socially optimal coverage, the Nash equilibrium driven by rational self-interest in most models leads to a vaccine coverage that is different (often lower) than the social optimum vaccine coverage (Figure 2).

Some models indicate that this free-riding manifestation of policy resistance can emerge relatively quickly upon introduction of a new immunization program, and that it can result in considerable instabilities in vaccine coverage $[19,42,8]$. For example, new generation vaccines for childhood immunization programs are launched in the United States every few years [3], and the success of the immunization program depends to some extent on how the population will respond to it, which may only partly be a function of demonstrated safety and efficacy of the vaccine. A recent game theoretical model describes how populations respond to a new pediatric infectious disease vaccine implemented through a universal mass vaccination program administered at a specified age every year [8]. This model predicts that, due to initially high infection prevalence, vaccine coverage remains reasonably high immediately after introduction, but can succumb to free-riding on herd immunity within 4-5 birth cohorts (years). These drops occurs sooner (within 2-3 birth cohorts) when the disease risk is low or vaccine efficacy is low. Moreover, due to instabilities in behaviour-incidence dynamics, vaccine coverage can vary considerably from one birth cohort to the next. The model also enables calculating the smallest vaccine risk tolerable for each birth cohort so that an individual make a rational decision of considering vaccination; this information may be useful for designing phase-III trials and phase-IV safety studies for vaccines [3].

Somewhat similar dynamics have been explored in the context of influenza vaccination [42]. Influenza management is one of the most significant current concerns for public health policy makers. While it is recommended that nearly $80 \%$ of individuals should get annual influenza vaccine, it is estimated that only $40-50 \%$ actually do so [23]. In response to the need to revaccinate for influenza every year, universal influenza vaccines conferring long-term immunity are being developed, and it is hoped that this might increase vaccine coverage [46]. However, a game theoretic model linking human cognition and memory for universal influenza immunization and influenza epidemiology forces us to consider the potential for policy resistance against universal influenza vaccines. This model predicts that a universal vaccine which provides short-term protection will on average increase the vaccine coverage, compared to the standard seasonal vaccine: short-term protection maintains risk communication of influenza among populations, resulting in stable vaccine coverage, which in turn creates small groups of free-riders and thus frequent but small-size epidemics. In contrast, a universal vaccine that provides longer-term 
protection may be counter-productive in some respects. Long-term protection creates large groups of free-riders who accept vaccination only after a severe epidemic occurs. Because of long-term immunity, individuals mostly free-ride, or accept vaccination only once in a large time-frame, and this results in drop of vaccine coverage after many years, in turn causing infrequent but very severe epidemics.

The imbalance between perceived and real risk and its negative effect on vaccine coverage is also reflected by several other examples of research. For example, population surveys have been used to parameterize game theoretical vaccinating behaviour models for influenza and human papillomavirus (HPV) vaccination [27, 4]. These models confirm that rational individual vaccinating decision-making would not allow populations to reach vaccine coverage levels that minimize disease prevalence in the population.

Another manifestation of policy resistance is individuals who vaccinate, but only after a period of delay. Delaying behaviour has been observed in some real-world immunization programs, and the game theoretical aspects of such behaviour have been explored for the case of pediatric infectious diseases [11] and pandemic influenza [12]. Using a game theoretic model of vaccination the authors have shown that relatively low disease incidence causes individuals to delay vaccination, for a year or two in the case of school-based programs for pediatric infectious diseases, or many weeks in the case of pandemic influenza. Naturally, delaying behaviour also hinders disease control and can cause subsequent incidence spikes.

\section{Policy reinforcement}

Models of vaccinating behaviour can also exhibit policy reinforcement, which can be defined as the tendency for interventions to be boosted by the system's response to the intervention. Instead of the negative feedback loop of policy resistance, where an increase in vaccine coverage tends to create a disincentive for further vaccination activity, the feedback loop in the case of policy reinforcement is positive, where increased vaccination activity stimulates still further vaccination activity.

One situation in which policy reinforcement can occur in such models is during the transient period when a new vaccine has been introduced and there is some social-learning process, whereby individuals adopt a vaccinator strategy only if they have learned that behaviour from someone else [7, 39, 20, 26, 9]. In that scenario, disease is initially widespread and there is little herd immunity, and so it is optimal for individuals to get vaccinated. At the same time, if individuals "sample" other individuals at some rate and only switch to being a vaccinator when they sample someone who is a vaccinator, then an increase in the abundance of vaccinators will lead to more instances of vaccinators being "sampled", and hence more opportunities for new vaccinators to be created. As a result, there is a virtuous cycle of increasing vaccine coverage, at least until herd immunity creates a disincentive large enough to outweigh the effect of increasing numbers of vaccinators. 
There are also other potential sources of policy reinforcement. For some infections (such as chickenpox), pathogenicity of an infection can increase with age, which can reverse the usual relationship between Nash equilibrium vaccine coverage and socially optimal vaccine coverage [34]. Unlike for a non-age structured model, vaccination against chickenpox may either help or harm the unvaccinated. While vaccination decreases the probability of being infected, it also shifts the average age at infection upward, and for an infection like chickenpox that can become more severe with age, this could end up harming the unvaccinated. It could also harm vaccinated individuals in whom the vaccine was not efficacious, leading to breakthrough infection. At high vaccine efficacy, while the infection probability for unvaccinated juveniles decreases with vaccination coverage, it actually increases for adults. For example, the infection probability for adults peaks at $18 \%$, when the background vaccine coverage is $77 \%$, though it starts declining as full herd immunity is approached at $92 \%$ coverage. For low vaccine efficacy, the infection probability peaks at $17 \%$, even if the entire population is vaccinated. The probability of breakthrough infection is also dependent on the vaccine coverage. For partial vaccine efficacy, the probability of breakthrough infection for both juveniles and adults increases with increasing vaccine coverage. With increasing vaccine coverage, fewer individuals are exposed to the disease thereby decreasing the total number of fully protected individuals relative to partially protected individuals. The probability of acquiring disease for partially protected individuals remains high when especially the coverage is low, though it drops very quickly as the herd immunity approached.

As a result of these effects, the Nash equilibrium vaccine coverage in this game theoretical chickenpox model can actually exceed the socially optimal vaccine coverage (Figure 3). From the perspective of the social optimum, this is not truly an example of policy reinforcement since the total health burden at the Nash equilibrium vaccine coverage still differs from the total health burden at the socially optimal vaccine coverage. However, from the perspective of universal vaccine coverage as a public health goal, we could view this as an example of policy reinforcement, since the emergence of high vaccine coverage is facilitated by the population response to the vaccine program.

\section{Outcome inelasticity}

The consequences of a vaccination game played out over the course of a single outbreak where the vaccine is new to the population has also been investigated, in the context of pandemic influenza vaccination [12]. This can lead to an effect termed outcome inelasticity, whereby a given outcome (such as prevalence of infection, or timing of an epidemic peak, or number of deaths, etc) is conserved across a given range of parameter values, due to nonlinear feedbacks in the model.

In a situation where a new vaccine has been introduced to a population experiencing an outbreak of an infectious disease, many individuals may opt to "wait and 
see" regarding vaccine risks. In particular, they may avoid vaccination until enough other individuals around them have vaccinated to convince them that the vaccine is safe. As a result, perceived vaccine risk decreases as a function of the number of individuals vaccinated to date. This amounts to a form of free-riding, where early vaccination by the entire population would have prevented the outbreak, but instead late vaccinators use early vaccinators as "guinea pigs". On the other hand, late vaccinators also accept an increased risk of being infected before they can get vaccinated. On top of this interaction, herd immunity also plays a role, with herd immunity provided by early vaccinators helping to protect late vaccinators or nonvaccinators.

In a game theoretical model capturing these strategies, it is possible to show how the strategic behaviour causes the timing of the pandemic peak to be strongly conserved across a broad range of plausible transmission rates, which is generally not possible without strategic behaviour. As the basic reproductive number $R_{0}$ increases it causes a more rapid development of the epidemic, which forces late vaccinators to vaccinate a little earlier, thus counteracting the effects of a higher $R_{0}$. The net effect is that the peak occurs at approximately the same week regardless of $R_{0}$, i.e., the outcome is inelastic with respect to $R_{0}$ (Figure 4) [12].

We note in passing that this model also exhibits policy reinforcement with respect to the initial perceived vaccine risk: an effort to decrease the initial perceived vaccine risk would supply benefits throughout the epidemic, because a lower initial perceived vaccine risk means more early vaccinators, which in turn means more "guinea pigs" from which late vaccinators can form an impression of vaccine safety, which means more late vaccinators and lower perceived vaccine risk later in the outbreak.

An example of outcome inelasticity with respect to total mortality in a population is observed in a non-game theoretical model of vaccinating behaviour for an infection transmitted through an evolving social contact network [36]. In this model, individuals who become infected have a probability $d_{\text {inf }}$ of dying, and this risk is part of the individual payoff functions. As the parameter $d_{\text {inf }}$ increases, the payoff for not vaccinating decreases, making vaccination a more appealing option. As a result, vaccine coverage increases as $d_{\text {inf }}$ increases, which counteracts the effects of a higher $d_{\text {inf }}$ to make the total number of deaths relatively constant across a range of values for $d_{\text {inf }}$.

\section{Outcome variability}

Outcome variability is a situation where, due to stochastic effects, qualitatively different outcomes are possible for different stochastic realizations of the same underlying model parameter distributions. This may occur at the boundary of basins of attraction for two steady states, and is a possible outcome of vaccinating behaviour models where some processes of social learning or imitation is present $[7,39,45,9]$.

For example, in a model of voluntary ring vaccination where individuals must weigh whether to get vaccinated given that their contact is an index case, outcome 
variability can occur when individuals are prone to imitate the decisions of other contacts of the index case [45]. For the same underlying model parameter distributions, some stochastic realizations result in the majority of contacts getting vaccinated, while other stochastic realizations result in the majority of contacts not getting vaccinated (Figure 5). As a result, the number of contacts who get infected can be either large or small. The implications of such social "clumpiness" in vaccine strategies due to imitation tendencies for the success of ring vaccination strategies are clear, since this can make the difference between control or no control of the outbreak.

Likewise, in a social learning model where the population is split evenly between vaccinators and non-vaccinators and where individuals tend to copy more successful strategies, the long-term outcome can be high vaccine coverage or low vaccine coverage depending on which basin of attraction the system is tipped into, based on stochastic effects.

\section{Discussion and Conclusion}

Human behaviour can be a key driver of infectious disease dynamics [25], particularly as it relates to vaccinating decisions [9]. Behaviour-incidence dynamics can thereby have significant implications for public health policy. The clash between Nash equilibrium and social optimum, where free-riding behaviour results in suboptimal vaccine uptake, is an example of policy resistance, which is the most commonly explored outcome of behaviour-incidence dynamics. However, here we have discussed other possible outcomes such as policy reinforcement, outcome inelasticity, and outcome variability. These effects nuance the standard "cartoon" of voluntary vaccination as a free-rider problem.

Such nuances can have important implications for vaccine policy. For example, the game theoretical model of chickenpox vaccination described in section 3 provides diverging recommendations for chickenpox vaccination policy in Israel versus the United States [34]. The authors suggest that external regulation such as subsidies for vaccination may be unnecessary or may even worsen the situation depending on the relationship between the Nash equilibrium and the social optimum. According to their analysis, subsidies or external regulation are required to achieve target coverage for the United States, whereas an information campaign on chickenpox awareness and vaccine safety is sufficient to optimize vaccine coverage in Israel.

Similarly, outcome inelasticity and outcome variability have implications for disease management. Reaction to a new vaccine during a pandemic outbreak and the potential for behavioural feedbacks to shape the epidemic curve have implications for risk messaging, prioritization of vaccination for high-risk groups, and logistics of vaccine rollout during a pandemic [12]. Similarly, ring vaccination may need to consider the social context (with specific emphasis on beliefs regarding vaccination) given the potential for social learning effects to influence local acceptance or rejection of a vaccine, and thus determine success of local containment $[24,45]$. 
The list of emergent dynamical effects of behaviour-incidence models we have provided here is not exhaustive, and more effects may come to light as the theory is developed. However, as the theory continues to develop, we hope that more efforts will be made to better validate these models against empirical data, and apply them to specific questions concerning real-world vaccine policies. As a result, we will better understand how to recognize, mitigate, and harness the emergent dynamics of behaviour-incidence systems in order to improve public health.

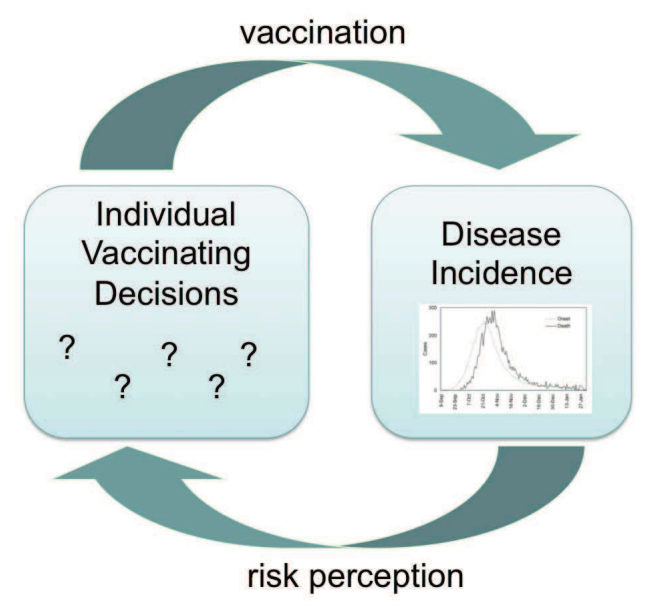

Fig. 1 Feedback loop arising from interactions between vaccinating behavior and disease incidence. Figure taken from [10].

Acknowledgements C.T.B. is supported by a Discovery Grant from the Natural Sciences and Engineering Research Council.

\section{References}

1. Anderson RM, May RM (1991) . Infectious Diseases ofHumans. Oxford Science Publications, Great Britain.

2. Auld C (2003) Choices, beliefs, and infectious disease dynamics. J. Health Econ. 22: 361377.

3. Ball L, Ball R, Gellin B 2004 Developing safe vaccines. In: Levine M, Kaper J, Rappuoli R, Liu M, Good M, eds. New generation vaccines. 127-144.

4. Basu S, Chapman GB, Galvani AP (2008) Integrating epidemiology, psychology, and economics to achieve HPV vaccination targets. Proc Natl Acad Sci. USA 105: 19018-19023.

5. Bauch CT, Earn DJD (2004) Vaccination and the theory of games. Proc Natl Acad Sci. USA 101:, 13391-13394.

6. Bauch CT, Galvani AP, Earn DJD (2003) Group interest versus self-interest in smallpox vaccination policy. Proc. Natl Acad. Sci. USA 100, 10564-10567. 


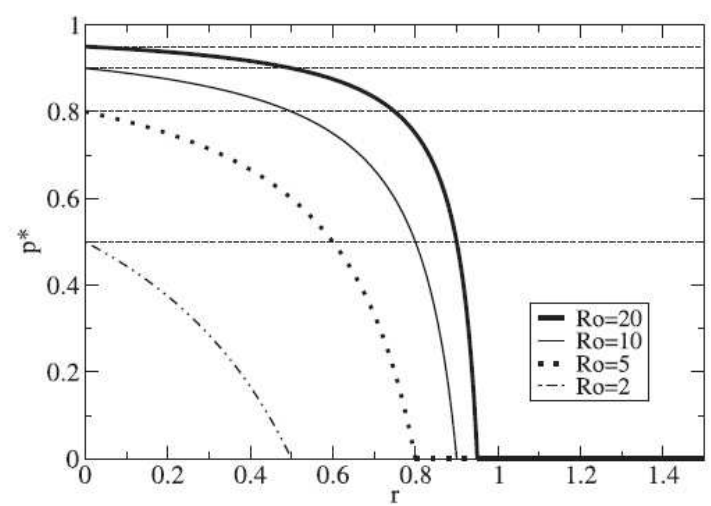

Fig. 2 Policy resistance in vaccination for paediatric infectious diseases. Vaccine coverage $p^{*}$ at the Nash equilibrium versus relative risk $r$, the ratio between risk of vaccination and risk of infection, for various values of $R_{0}$. Dashed horizontal lines demarcate the critical coverage level that eliminates the disease from the population. Figure taken from [5].
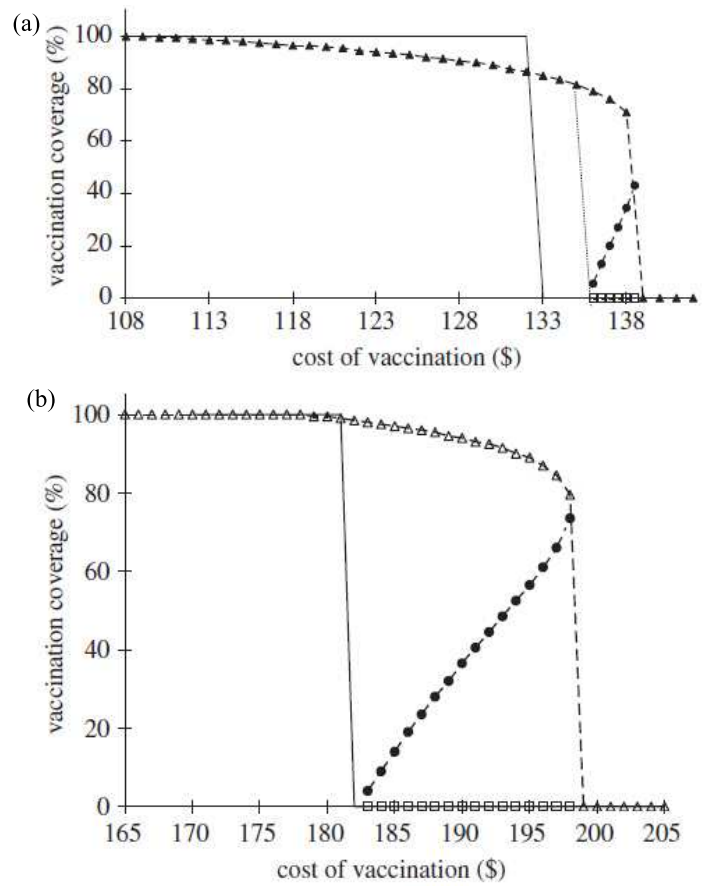

Fig. 3 Policy enhancement in chickenpox vaccination. Nash equilibria versus social optimum for a range of cost of vaccinations in (a) USA and (b) Israel. Depending on the vaccination cost, there are three Nash equilibria indicated by triangles, squares and filled circles. Solid line (without symbols) indicates the social optima as a function of cost of vaccination. Figure taken from [34]. 


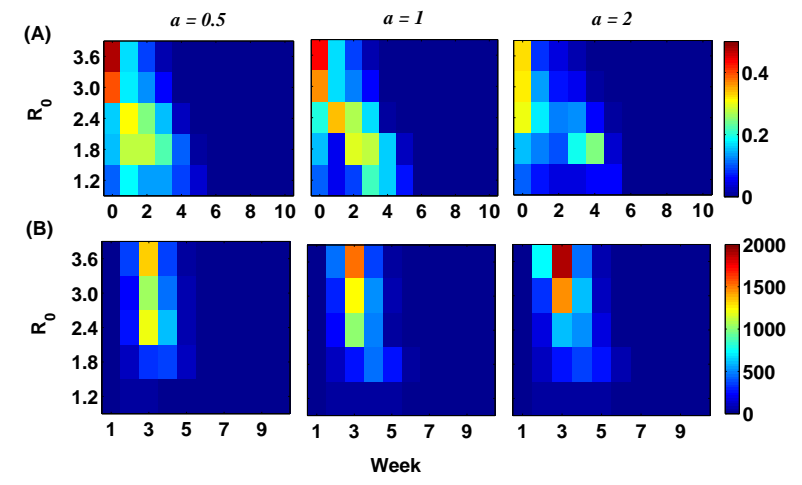

Fig. 4 Outcome inelasticity in pandemic influenza vaccination. (a) Nash equilibrium vaccine coverage and (b) resulting influenza incidence, at different $R_{0}$ values and different values of the parameter $a$ governing perceived risk evolution during the pandemic, over each week of the outbreak. Figure taken from [12].
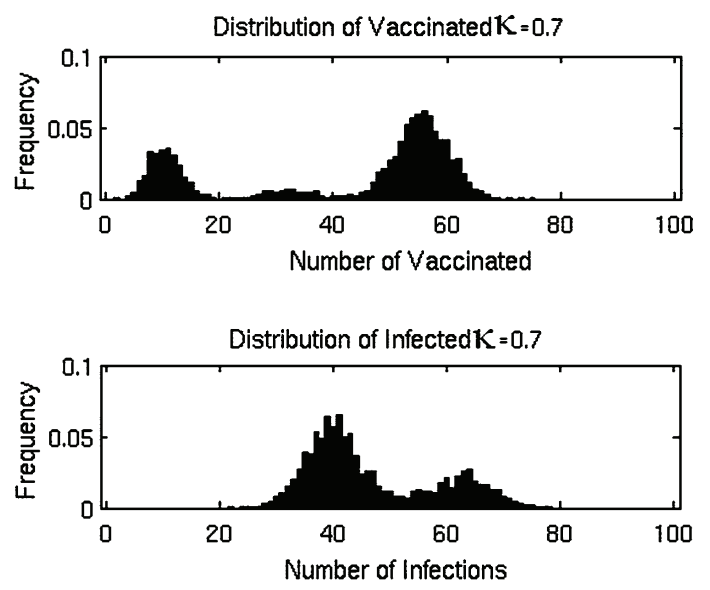

Fig. 5 Outcome variability in voluntary ring vaccination. The distribution across many stochastic realizations of the number vaccinated (top) and the number of secondary infections (bottom) in 100 neighbours of an index case, for imitation strength $\kappa=0.7$. Figure adapted from [45]. For details see this reference. 
7. Bauch CT (2005) Imitation dynamics predict vaccinating behaviour. Proc R Soc B 272: 16691675 .

8. Bauch CT, Bhattacharyya S, Ball RF (2010) Rapid Emergence of Free-Riding Behavior in New Pediatric Immunization Programs, PLoS ONE 5(9), e12594

9. Bauch CT, Bhattacharyya S (2012) Evolutionary game theory and social learning can determine how vaccine scares unfold, PLos Comp Biol., 8(4):e1002452.

10. Bhattacharyya S, Bauch CT, (2012) Mathematical models of the interplay between individual vaccinating decisions and disease dynamics: a need for closer integration of models and data, Human Vaccine and Immunotherqpeutics, 8(6).

11. Bhattacharyya S, Bauch CT, (2010) A game dynamic model for delayer strategies in vaccinating behaviour for pediatric infectious diseases. J Theor Biol. 267: 276-282.

12. Bhattacharyya S, Bauch CT, (2011) "Wait and see" vaccinating behaviour during a pandemic: A game theoretic analysis. Vaccine 29: 5519-5525.

13. Bonanni P (1999) Demographic impact of vaccination: a review, Vaccine 17: 120-125

14. Brito DL, Sheshinski E, Intriligator MD (1991) Externalities and compulsory vaccinations. J Public Econ., 45: 69-90.

15. Coelho FC, Codec OCT (2009) Dynamic modeling of vaccinating behavior as a function of individual beliefs. PLoS Comput Biol 5: e1000425.

16. Colgrave $\mathbf{J}$ (2006) State of immunity: the politics of vaccination in twentieth-century America. Berkeley, CA: University of California Press.

17. Diel R, Rappenhoener B, Schneider S (2000) Cost-effectiveness of hepatitis A immunization of children and adolescents in Germany. Eur J Health Econ. 2(3): 96-103.

18. d'Onofrio A, Manfredi P, Salinelli E (2008) Fatal SIR diseases and rational exemption to vaccination. Math Med Biol. 25: 337-357.

19. d'Onofrio A, Manfredi P, Salinelli E (2007) Vaccinating behaviour, information, and the dynamics of SIR vaccine preventable diseases. Theor. Popul. Biol. 71: 301-317.

20. d'Onofrio A, Manfredi P, Poletti P (2011) The impact of vaccine side effects on the natural history of immunization programmes: an imitation-game approach. J Theor Biol. 273(1):6371.

21. Fenner FDA, Henderon DA, Arita I et al. (1988) Smallpox and its eradication. Geneva: World Health Organization.

22. Fine PE, Clarkson JA (1986) Individual versus public priorities in the determination of optimal vaccination policies. Am J Epidemiol. 124: 1012-1020.

23. Fiore AE, Shay DK, Broder K et al. (2009) Prevention and Control of Seasonal Influenza with Vaccines Recommendations of the Advisory Committee on Immunization Practices (ACIP), 2009. Book Prevention and Control of Seasonal Influenza with Vaccines Recommendations of the Advisory Committee on Immunization Practices (ACIP), 1-52.

24. Funk S, Gilad E, Jansen VAA (2010) Endemic disease, awareness, and local behavioural response. J Theor Biol. 264: 501-509.

25. Funk S, Salath M, Jansen VAA (2010) Modelling the influence of human behaviour on the spread of infectious disease: A review. J R Soc Interface, doi:10.1098/rsif.2010.0142.

26. Fu F, Rosenbloom DI, Wang L, Nowak MA (2011) Imitation dynamics of vaccination behaviour on social networks. Proc R Soc B 278: 42-49.

27. Galvani AP, Reluga TC, Chapman GB (2007) Long-standing influenza vaccination policy is in accord with individual self-interest but not with the utilitarian optimum. Proc Natl Acad Sci. USA 104: 5692-5697.

28. Gharbieh EA, Fahmy S, Rasool BA, Khan S (2010) Influenza Vaccination: Healthcare Workers Attitude in Three Middle East Countries. Int J Med Sci 7(5): 319-325.

29. Greenhalgh D (1986) Optimal control of an epidemic by ring vaccination. Commun Stat Stoch Models, 2: 339-363.

30. Hardin G (1968) The tragedy of the commons. Science 162: 1243-1248.

31. Jansen VA, Stollenwerk N, Jensen HJ, Ramsay ME, Edmunds WJ, Rhodes CJ (2003) Measles outbreaks in a population with declining vaccine uptake. Science 301: 804.

32. Keeling MJ, Woolhouse MEJ, May RM et al. (2003) Modelling vaccination strategies against foot-and-mouth disease. Nature, 421: 136-142. 
33. Lam PP, Chambers LW, MacDougall DMP et al. (2010) Seasonal influenza vaccination campaigns for health care personnel: systematic review. CAMJ, 182(12): E542-E548.

34. Liu J, Kochin BF, Tekle YI, Galvani AP (2012) Epidemiological game-theory dynamics of chickenpox vaccination in the USA and Israel', Interface 9(66): 68-76.

35. Madjid M. et al. (2009) Factors Contributing to Suboptimal Vaccination against Influenza, Tex Heart Inst J 36(6): 546-52.

36. Morsky B, Bauch CT (2012) Outcome inelasticity and outcome variability in behaviorincidence models: an example from an SIR infection on a dynamic network. Submitted.

37. Muller J, Schonfisch B, Kirkilionis M (2000). Ring vaccination. J Math Biol., 41: 143-171.

38. Philipson T (1996) Private vaccination and public health: an empirical examination for US measles. J. Hum. Resour. 31, 611-630.

39. Reluga TC, Bauch CT, Galvani AP (2006) Evolving public perceptions and stability in vaccine uptake. Math Biosci. 204: 185-198.

40. Sterman JD (2006) Learning from Evidence in a Complex World. Am J Pub Health, 96: 505514.

41. Toma B, Moutou F, Dufourc B et al. (2002). Ring vaccination against foot-and-mouth disease. Comp Immunol Microbiol Infect Dis, 25, 365-372.

42. Vardavas R, Breban R, Blower S (2010) A universal long-term flu-vaccine may not prevent severe epidemics. Vardavas et al. BMC Research Notes 3: 92.

43. Von Neumann J, Morgenstern O (1944) Theory of games and economic behavior. Princeton, NJ. Princeton University Press.

44. Weibull JW (1995) Evolutionary game theory. Cambridge, MA: MIT Press.

45. Wells CR, Tchuenche JM, Meyers L et al (2011) Impact of Imitation Processes on the Effectiveness of Ring Vaccination, doi 10.1007/s11538-011-9646-4

46. Wilkinson E (2008) Universal flu vaccine tests start. Book Universal flu vaccine tests start. 